\title{
Diabetes and Hyperthyroidism: Is There a Causal Link?
}

\author{
Sang Yong Kim \\ Division of Endocrinology, Department of Internal Medicine, Chosun University College of Medicine, Gwangju, Korea
}

Diabetes mellitus (DM) and thyroid dysfunction (TD) are the two most common endocrine disorders in clinical practice. It is well known that Hashimoto's thyroiditis and Graves' disease are autoimmune disorders that constitute the most prevalent forms of TD [1]. It is also known that type $1 \mathrm{DM}$ occurs due to autoimmune destruction of pancreatic $\beta$-cells, leading to absolute insulin deficiency [2]. The combination of these types of TD and type $1 \mathrm{DM}$, as autoimmune-induced endocrine disorders, is termed polyglandular autoimmune syndrome [3]. Beyond these relationships between autoimmune-induced endocrine disorders, several studies have demonstrated that TD is closely related to DM. TD is more common in patients with type $2 \mathrm{DM}$ than in the general population and can adversely influence their metabolic control. The overall prevalence of TD in patients with type $2 \mathrm{DM}$ in several countries has been reported to range from $4 \%$ to $20 \%[4,5]$. It is well known that both hyperthyroidism and hypothyroidism can change glucose and lipid metabolism. Metabolic changes in patients with hyperthyroidism mainly result from increased insulin resistance, because excess thyroid hormone increases endogenous glucose production and insulin requirements and reduces hepatic insulin sensitivity [6]. Therefore, when hyperthyroidism occurs in patients with DM, they are at an elevated risk of severe hyperglycemia and poor glycemic control. However, although the association between hyperthyroidism and glucose metabolism is well documented, few studies have prospectively investigated the relationship between TD and new-onset DM. Moreover, the studies that have addressed this issue have predominantly investigated the relation-

Received: 1 December 2021, Accepted: 7 December 2021

Corresponding author: Sang Yong Kim

Division of Endocrinology, Department of Internal Medicine, Chosun University

College of Medicine, 309 Pilmun-daero, Dong-gu, Gwangju 61452, Korea

Tel: +82-62-220-3011, Fax: +82-62-223-3316, E-mail: diabetes@chosun.ac.kr ship between hypothyroidism and the incidence of DM [7,8]. In this respect, it is necessary to investigate the risk of DM in patients with hyperthyroidism and to establish a screening program accordingly.

In this issue of Endocrinology and Metabolism, Song et al. [9] published a study investigating the risk of DM in patients with long-standing Graves' disease using retrospective data from the Korean National Health Insurance Service database. Long-standing Graves' disease was defined as anti-thyroid drug (ATD) treatment for more than 24 months after the diagnosis of hyperthyroidism. The patients were also subclassified into a group that maintained ATD for more than 12 months after initial treatment for 24 months and a group that received radioactive iodine ablation (RIA) therapy after initial treatment. The authors reported that the hazard ratio for DM occurrence was 1.18 in patients with hyperthyroidism after adjustment compared to the control group. The risk of DM increased with an increased duration of ATD treatment, as well as in the RIA therapy group. From these results, we can infer that hyperthyroidism increases the incidence of DM by causing changes in blood glucose metabolism, especially in patients receiving long-term ATD therapy or RAI therapy. Notably, the risk of DM was particularly prominent in patients with a relatively low body mass index (BMI) and those without dyslipidemia. Because high BMI and dyslipidemia are regarded as conventional risk factors for DM, it is noteworthy that the risk of DM increased in patients with a low BMI and a normal lipid profile in this analysis. Therefore, this result means that hyperthyroidism and hypothyroidism may

Copyright $\odot 2021$ Korean Endocrine Society

This is an Open Access article distributed under the terms of the Creative Commons Attribution Non-Commercial License (https://creativecommons.org/ licenses/by-nc/4.0/) which permits unrestricted non-commercial use, distribution, and reproduction in any medium, provided the original work is properly cited. 
be linked to the occurrence of DM through different mechanisms. It is also necessary to observe changes in blood glucose more carefully in patients with hyperthyroidism who do not have traditional risk factors for DM. However, current guidelines for the management of hyperthyroidism do not recommend serum glucose monitoring or screening for DM [10,11]. Regular tests for thyroid function or thyroid auto-antibody are recommended in patients with type $1 \mathrm{DM}$, but precautions for monitoring thyroid function in patients with type $2 \mathrm{DM}$ or routine screening for DM in patients with long-term use of ATDs or RAI therapy are not yet included in the recommendations. More prospective studies, including randomized trials, should be conducted in order to update the recommendations.

Due to the nature of health insurance databases, information on thyroid function is limited. There is a possibility that the patients enrolled in that study may have had more serious TD because they needed longer-term treatment and even RAI treatment. Thyroid hormones exert various effects on the regulation of glucose and lipid metabolism, and these effects are mediated by both central and peripheral actions [12]. Therefore, various studies to evaluate the relationship between the severity of hyperthyroidism and the risk of DM are needed in the future. Furthermore, as the authors pointed out, it was not possible to verify the accuracy of diagnoses of Graves' disease because patients with Graves' disease were identified based on International Classification of Diseases, Tenth Revision codes. Although Graves' disease accounts for the majority of hyperthyroidism in Korea, there is a possibility that due to clinicians' lack of experience, hyperthyroidism from other causes may have been mistaken for Graves' disease, resulting in incorrect coding. Nevertheless, this study is the first longitudinal study to assess the incidence of DM in patients with long-standing Graves' disease. Since the studies conducted to date are very limited and fragmentary, this study provides valuable data on the risk of DM in patients with Graves' disease through a long-term study of a national database. In particular, since most guidelines for hyperthyroidism recommend ATD therapy within 18 months, the data from this study can provide evidence supporting regular blood glucose monitoring as part of the evaluation of side effects when using ATDs for more than 24 months. In addition, we should monitor glucose levels more carefully in patients who receive RAI therapy after the failure of long-term ATD therapy. Many questions remain to be clarified. It has yet to be established whether long-standing Graves' disease itself increases the risk of DM, whether the risk of DM varies depending on the severity of hyperthyroidism, or whether long-term ATD use or
RAI therapy itself affects the incidence of DM. Based on this study, we hope that these questions will be solved by additional creative and prospective studies in the future.

\section{CONFLICTS OF INTEREST}

No potential conflict of interest relevant to this article was reported.

\section{ORCID}

Sang Yong Kim https://orcid.org/0000-0002-3902-622X

\section{REFERENCES}

1. Biondi B, Kahaly GJ, Robertson RP. Thyroid dysfunction and diabetes mellitus: two closely associated disorders. Endocr Rev 2019;40:789-824.

2. American Diabetes Association. 2. Classification and diagnosis of diabetes: standards of medical care in diabetes-2018. Diabetes Care 2018;41(Suppl 1):S13-27.

3. Kahaly GJ. Polyglandular autoimmune syndromes. Eur J Endocrinol 2009;161:11-20.

4. Papazafiropoulou A, Sotiropoulos A, Kokolaki A, Kardara M, Stamataki P, Pappas S. Prevalence of thyroid dysfunction among Greek type 2 diabetic patients attending an outpatient clinic. J Clin Med Res 2010;2:75-8.

5. Akbar DH, Ahmed MM, Al-Mughales J. Thyroid dysfunction and thyroid autoimmunity in Saudi type 2 diabetics. Acta Diabetol 2006;43:14-8.

6. Potenza M, Via MA, Yanagisawa RT. Excess thyroid hormone and carbohydrate metabolism. Endocr Pract 2009; 15: 254-62.

7. Gronich N, Deftereos SN, Lavi I, Persidis AS, Abernethy DR, Rennert G. Hypothyroidism is a risk factor for new-onset diabetes: a cohort study. Diabetes Care 2015;38:165764.

8. Chaker L, Ligthart S, Korevaar TI, Hofman A, Franco OH, Peeters RP, et al. Thyroid function and risk of type 2 diabetes: a population-based prospective cohort study. BMC Med 2016;14:150.

9. Song E, Koo MJ, Noh E, Hwang SY, Park MJ, Kim JA, et al. Risk of diabetes in patients with long-standing Graves' disease: a longitudinal study. Endocrinol Metab (Seoul) 2021;36:1277-86.

10. Kahaly GJ, Bartalena L, Hegedus L, Leenhardt L, Poppe K, 
Pearce SH. 2018 European Thyroid Association guideline for the management of Graves' hyperthyroidism. Eur Thyroid J 2018;7:167-86.

11. Moon JH, Yi KH. The diagnosis and management of hyper- thyroidism in Korea: consensus report of the Korean Thyroid Association. Endocrinol Metab (Seoul) 2013;28:275-9.

12. Brent GA. Mechanisms of thyroid hormone action. J Clin Invest 2012;122:3035-43. 